\title{
Histiocytosis: a review focusing on neuroimaging findings
}

\author{
Histiocitoses: uma revisão focada em achados de neuroimagem \\ Larissa Barcessat Gabbay ${ }^{1}$ Cláudia da Costa Leite ${ }^{2,3}$, Ranieli Saraiva Andriola ${ }^{1}$, Paula da Cunha Pinho", \\ Leandro Tavares Lucato ${ }^{4}$
}

\begin{abstract}
Objective: Histiocytosis is a systemic disease that usually affects the central nervous system. The aim of this study is to discuss the neuroimaging characteristics of Langerhans cell histiocytosis $(\mathrm{LCH})$, the most common of these diseases; and the non-Langerhans cells histiocytosis (NLCH), which includes entities such as hemophagocytic syndrome, Erdheim-Chester and Rosai-Dorfman diseases. Method: Literature review and illustrative cases with pathologic confirmation. Results: In LCH, the most common findings are 1) osseous lesions in the craniofacial bones and/or skull base; 2) intracranial, extra-axial changes; 3) intra-axial parenchymal changes (white and gray matter); 4) atrophy. Among the $\mathrm{NLCH}$, diagnosis usually requires correlation with clinical and laboratory criteria. The spectrum of presentation includes intraparenchymal involvement, meningeal lesions, orbits and paranasal sinus involvement. Conclusion: It is important the recognition of the most common imaging patterns, in order to include LCH and NLCH in the differential diagnosis, whenever pertinent.
\end{abstract}

Keywords: Langerhans cells histiocytosis, non-Langerhans cells histiocytosis, Rosai-Dorfman, hemophagocytic syndrome, ErdheimChester.

RESUMO

Objetivo: Histiocitose é uma doença sistêmica que, frequentemente, acomete o sistema nervoso central. Este trabalho propõe discutir as características de neuroimagem da histiocitose de células de Langerhans (HCL), mais comum; e as histiocitoses não-Langerhans (HNL), como a síndrome hemofagocítica, doenças de Erdheim-Chester e Rosai-Dorfman. Método: Revisão de literatura, com demonstração de casos confirmados por anatomopatológico. Resultados: Na HCL os achados mais comuns são: 1) lesões ósseas craniofaciais e da base do crânio; 2) lesões intracranianas, extra-axiais; 3) acometimento parenquimatoso intra-axial na substância cinzenta ou branca; 4) atrofia. Dentre as HNL, o diagnóstico, em geral, exige correlação com critérios clínico-laboratoriais. O espectro de apresentação das mesmas inclui acometimento intraparenquimatoso, lesões meníngeas, envolvimento orbitário e das cavidades paranasais. Conclusão: É importante o reconhecimento dos padrões de imagem mais comuns, para que HCL e HNL sejam incluídas no diagnóstico diferencial, sempre que pertinente.

Palavras-chave: Histiocitose de células de Langerhans, histiocitoses não-Langerhans, Rosai-Dorfman, síndrome hemofagocítica, Erdheim-Chester.

Histiocytosis is a systemic disease that usually affects many organs, including the central nervous system (CNS). This group of disorders is characterized by an uncontrolled histiocytic proliferation, usually of unknown etiology, which triggers an immunologically mediated inflammatory process. Eventually, external agents such as infectious processes or neoplasms may be considered as the trigger factors.

The aim of this study is to discuss the neuroimaging characteristics of Langerhans cell histiocytosis, the most common of these diseases, and also the less common ones, grouped under the category non-Langerhans cells histiocytosis. This last group includes entities such as hemophagocytic syndrome, Rosai-Dorfman and Erdheim-Chester diseases.

Some of these diseases share imaging findings such as extra-axial lesions, hypothalamic-pituitary axis involvement and skull involvement. However, as it will be clear in the following sections, there are some differences that can help to differentiate between them.

\footnotetext{
${ }^{1}$ Instituto de Radiologia, Hospital das Clínicas, Faculdade de Medicina, Universidade de São Paulo, Sao Paulo SP, Brazil;

2Departamento de Radiologia, Faculdade de Medicina, Universidade de São Paulo, Sao Paulo SP, Brazil;

${ }^{3}$ Department of Radiology, University of North Carolina at Chapel Hill, Chapel Hill NC, USA;

${ }^{4}$ Centro de Diagnósticos Brasil (CDB), Sao Paulo SP, Brazil.

Correspondence: Larissa Barcessat Gabbay; Av. Dr. Enéas de Carvalho Aguiar, s/nº / Rua 1 Cerqueira César; 05403-900 São Paulo SP, Brasil;

E-mail: larissagabbay@hotmail.com

Conflict of interest: There is no conflict of interest to declare.

Received 27 March 2014; Received in final form 03 April 2014; Accepted 23 April 2014.
} 


\section{LANGERHANS CELL HISTIOCYTOSIS}

Langerhans cell histiocytosis (LCH) is a rare systemic granulomatous disease of the dendritic system with a variable clinical course that may be encountered at any age and may affect almost any organ ${ }^{1}$.

CNS involvement in LCH has been recognized since the early reports of the disease because of the frequent involvement of the cranial bones and the hypothalamic-pituitary region (HPR) with diabetes insipidus (DI) as key manifestation. Less frequently, granulomatous lesions in the meninges, the choroid plexus, the pineal gland, or the cerebral parenchyma can be encountered ${ }^{2}$.

In the past decades, a wide variety of other CNS findings have been described since the advent of magnetic resonance imaging (MRI), with or without associated clinical neuroendocrine findings.

The second most frequent presentation of $\mathrm{CNS} \mathrm{LCH}$, excluding HPR disease, is a combination of pathologic changes in the cerebellum, basal ganglia, and/or pons with characteristic MRI patterns ${ }^{3}$. Results of histopathologic examination of tissue from cerebellar biopsies and autopsies of patients with such MR imaging changes revealed neuron loss and axonal degeneration along with a profound T-cell inflammation. Thus, the described MR imaging signal intensity abnormalities were interpreted as indicative of a neurodegenerative process $(\mathrm{ND})^{4}$.

Apart from the extension of these so-called "neurodegenerative" lesions, there are 2 other types of parenchymal changes: (a) the frequently found dilated Virchow- Robin spaces (VRS) and (b) the rare leukoencephalopathy pattern².

\section{Clinical features}

The clinical presentation ranges from a single bone lesion to widespread multiorgan involvement. The course is unpredictable, with a spectrum of spontaneous regression, chronic recurrences for years, or a rapidly fatal deterioration ${ }^{5}$.

Intracranial lesions have been observed during the course of disease in patients with a history of proved $\mathrm{LCH}$ and also as the first and presenting LCH manifestations, in which case they can cause considerable diagnostic challenge ${ }^{3}$.

Four groups of patients can be clinically distinguished: 1) those with a disorder of the hypothalamic-pituitary system; 2) patients presenting with site-dependent symptoms from space-occupying lesions, such as seizures; 3) those who exhibit a neurologic dysfunction mostly following a cerebellar-pontine pathway involvement, including reflex abnormalities, ataxia, intellectual impairment, tremor, or dysarthria with variable progression to severe CNS deterioration; and 4) patients who present with an overlap of the symptoms mentioned previously 6 .

\section{Histopathologic findings}

Typical granulomas consist of an accumulation of Langerhans cells, indeterminate and interdigitating cells, macrophages and T-lymphocytes, and variable numbers of multinucleated giant cells and eosinophils. Birbeck granules are present, and CDla is immunoreactive ${ }^{4}$.

\section{MRI findings and differential diagnosis}

In $\mathrm{LCH}$, the wide spectrum of cranial and intracranial MRI findings include: 1) osseous lesions in the craniofacial bones and/or skull base with or without soft-tissue extension; 2) intracranial, extra-axial changes (involvement of hypothalamic-pituitary region, meninges, and other circumventricular organs, including the pineal gland, choroid plexus, and ependyma); 3) intra-axial parenchymal changes (white matter and gray matter) with a predominance of a symmetric neurodegenerative pattern and; 4) cerebral atrophy ${ }^{3}$.

The most frequently encountered manifestation of $\mathrm{LCH}$ in patients with localized or multisystem disease is the granulomatous osseous lesion, most commonly affecting the skull (Figure 1). These lesions are often osteolytic with a sclerotic margin. Soft-tissue extension may be observed. The differential diagnosis of lytic osseous lesions of the calvaria in childhood includes mostly neoplasm (metastases), infectious/inflammatory (sarcoidosis, tuberculosis) and leptomeningeal cyst. Lytic osseous lesions of the craniofacial bones may also be observed in fibrous dysplasia, rhabdomyosarcoma, or severe mastoiditis? .

The most common CNS manifestation in LCH is infiltration of the hypothalamic pituitary region by LCH granuloma (Figure 2), frequently leading to diabetes insipidus and anterior pituitary hormone deficiency ${ }^{4}$. The characteristic features consist of enlargement of the pituitary stalk with potential progression to space-occupying tumors extending to the pituitary and hypothalamus. Given the association of DI with LCH, there can be also typically loss of the physiologic hyperintense signal of the posterior pituitary

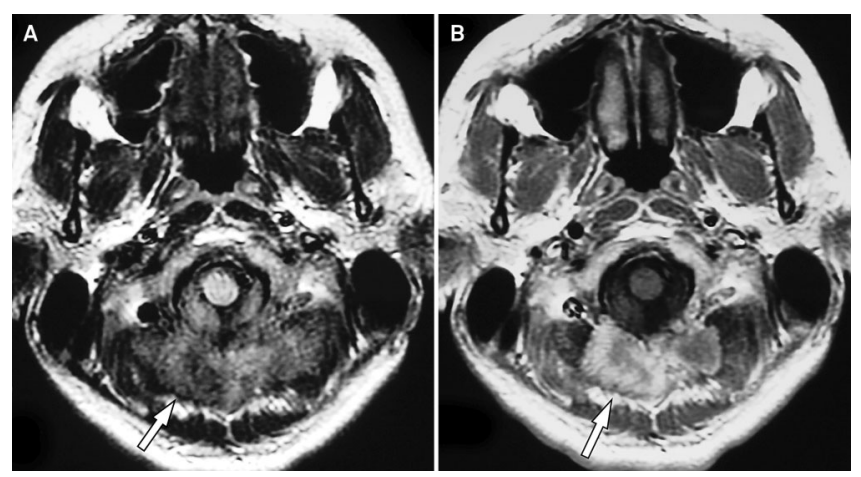

Figure 1. Langerhans cell hystiocitosis in a 19-year-old patient. MR images in the axial plane, sequences FLAIR (A) and postcontrast T1WI (B) disclose a lesion in the occipital bone, with intermediate signal intensity in FLAIR (arrow in A) and intense contrast enhancement (arrow in B). 

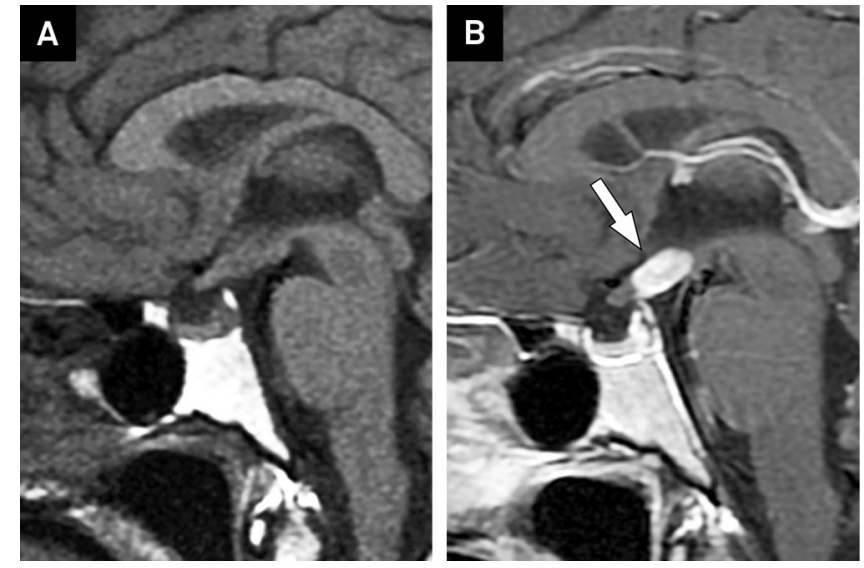

Figure 2. Langerhans cell hystiocitosis in a patient with diabetes insipidus. Sagittal T1WI image demonstrates a thickened pituitary infundibulum and absence of the normal T1-hyperintense posterior pituitary lobe (A). Enhanced sagittal T1WI image demonstrates intense contrast enhancement of the thickened infundibulum (B and arrow)

on T1-weighted images, which correlates with the loss of antidiuretic hormone-containing granules. In addition, threadlike narrowing of the infundibulum with a maximum width less than $1 \mathrm{~mm}$ can be seen ${ }^{2}$.

The absence of hyperintensity in the posterior pituitary and the presence of an infundibular or hypothalamic mass are nonspecific findings. Differential diagnosis of these findings is broad, including mostly: intracranial tumors (germinomas), granulomatous diseases (sarcoidosis, Wegener granulomatosis), leukemia, and autoimmune infundibuloneurohypohysitis ${ }^{8}$.

The high frequency of pineal cysts and enlarged pineal glands in patients with LCH remains a nonspecific but striking observation; this may reflect direct pineal infiltration by LCH or hyperplasia of the gland. The co-existing changes in the pituitary and pineal regions might be caused by their
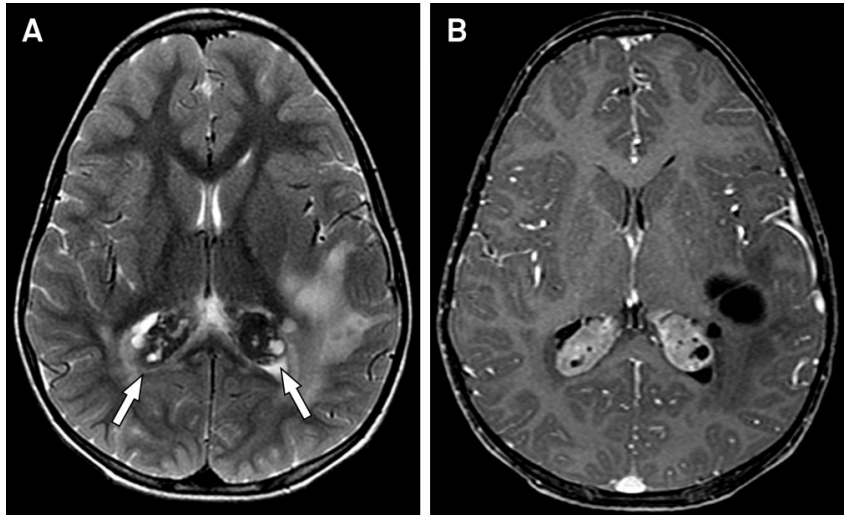

Figure 4. Langerhans cell hystiocitosis in a 5-year-old boy with exophthalmos, polyuria, polydipsia, and skin lesions. MR shows solid expansive lesions in the choroid plexus of both ventricular atria, characterized by hypointensity on $\mathrm{T} 2 \mathrm{WI}$ (arrows in A) and intense contrast enhancement in corres ponding T1WI (B). Notice some white matter edema near the atrium of the left lateral ventricle, associated to cystic areas more evident in the postcontrast T1WI.

functional interactions, because both structures belong to the circumventricular organs that are located outside the blood brain barrier?.

Other space occupying tumoral lesions occur rarely in the meninges (Figure 3), choroid plexus (Figure 4), and in the brain parenchyma (Figure 5). These lesions are typically characterized by signal intensity corresponding to soft tissue, presenting intermediate intensity on T1-weighted images (T1WI) and T2-weighted images (T2WI) with moderate or marked homogeneous contrast-enhancement).

Choroid-plexus lesions, which might otherwise mimic papillomas, are characterized by a marked hypointensity on T2WI (Figure 4). Differential diagnosis of intraparenchymal CNS lesions includes germ cell tumor, craniopharyngioma, sarcoidosis, or Rosai-Dorfman disease ${ }^{7}$.
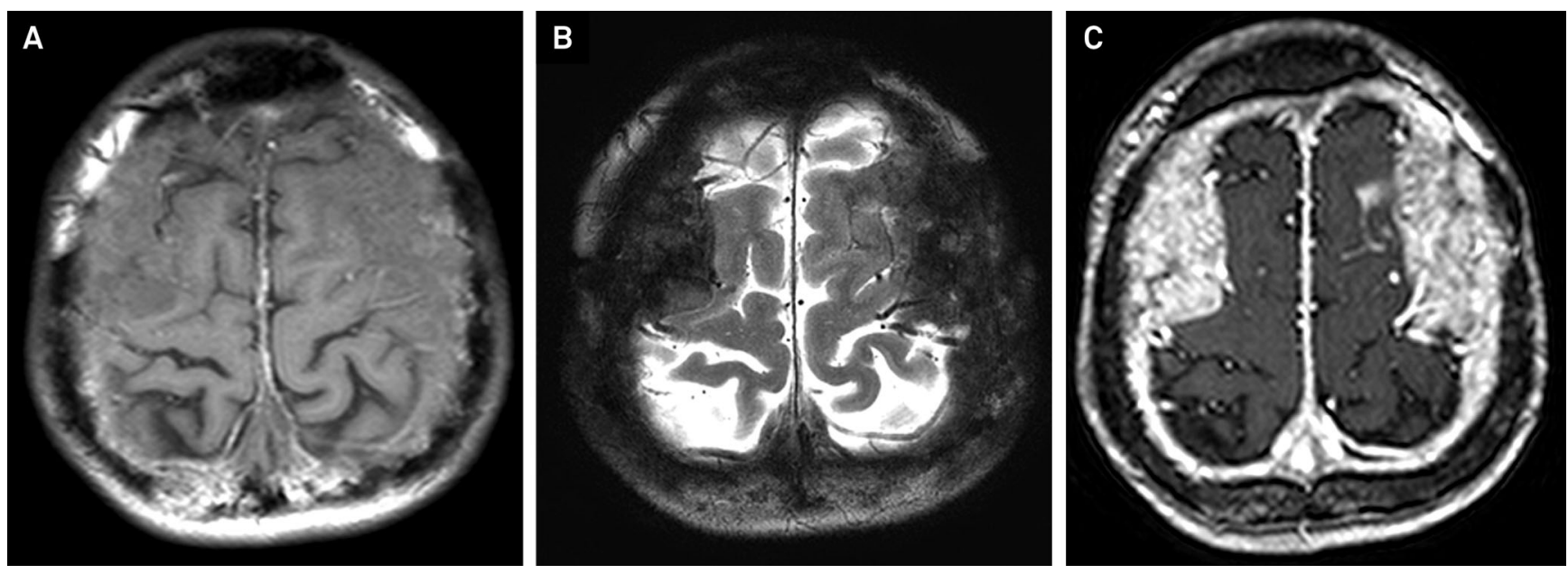

Figure 3. Patient with systemic Langerhans cell hystiocitosis. Axial T1WI (A), T2WI (B) and postcontrast T1WI (C). Extra-axial, bilateral space-occupying lesions originating from the meninges (mainly dura mater) in the high convexity. There is marked hypointensity in T2WI and intense contrast enhancement. 

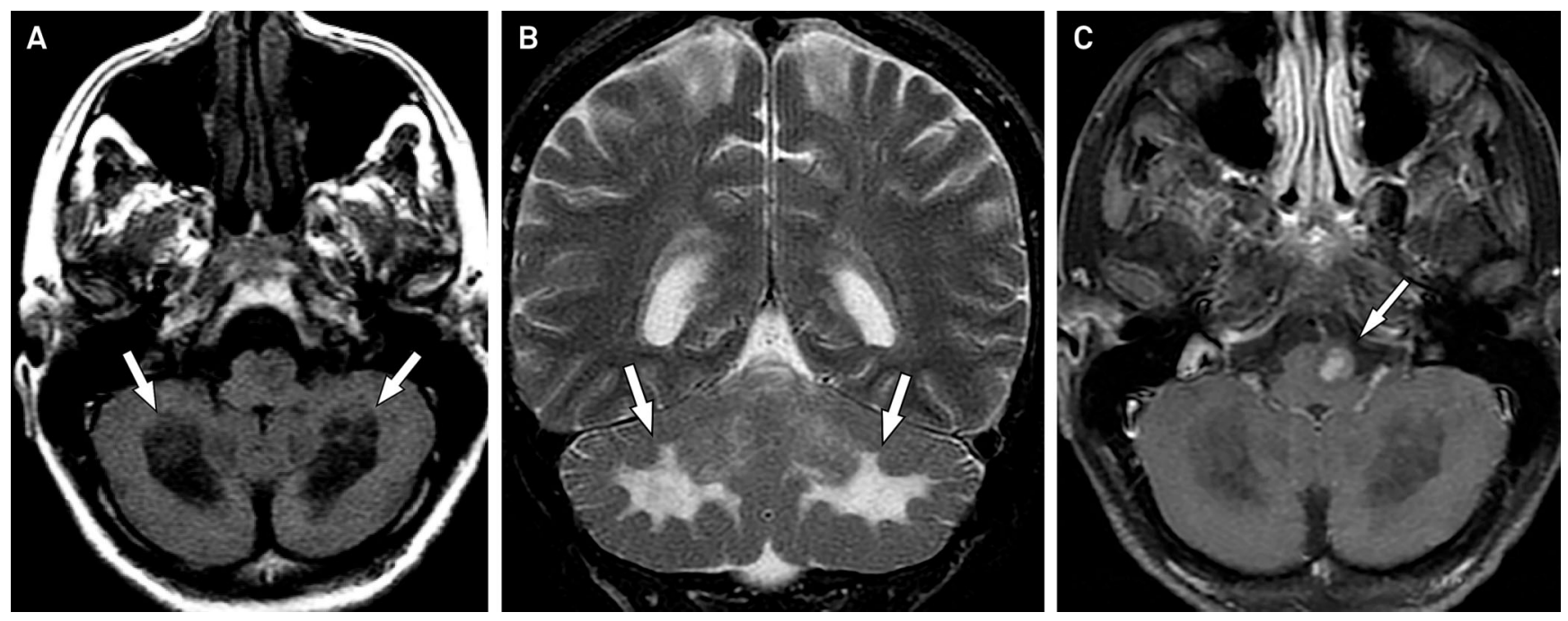

Figure 5. Langerhans cell hystiocitosis patient with ataxia, diabetes insipidus and behavioral disturbances. Axial T1WI shows hypointense lesions in the deep white matter of both cerebellar hemispheres (A and arrows). Coronal T2WI discloses the hyperintensity of these lesions (B and arrows). Postcontrast axial T1WI demonstrates that the cerebellar lesions do not enhance; however, there is nodular enhancement of a lesion in the anterior medulla ( $\mathrm{C}$ and arrow).

In addition to granulomatous lesions, intracerebral abnormalities also include progressive neurodegenerative changes (ND-LCH) within the cerebellum, basal ganglia and pons (Figure 5).

Neurodegenerative signal intensity abnormalities are a rare consequence of $\mathrm{LCH}$. The prevalence of radiologic ND-LCH in pediatric patients with LCH is uncertain and ranges in different reports between $4 \%$ and $10 \%$. Recent studies indicate that the radiologic ND-LCH is more frequently detected in patients with craniofacial lesions or neuroendocrine problems ${ }^{10}$.

These degenerative changes are characterized by symmetrically increased signal intensity in T2-weighted sequences within the cerebellar gray matter extending into adjacent white matter and having hypo- or hyperintensity on T1-WI. Pontine lesions have been reported as T2 hyperintense signal abnormalities in the tegmentum or pyramidal tracts. In the basal ganglia, the abnormalities consist of hyperintensities on T1-weighted images and variable signal intensities on T2-weighted images (Figure 6). These lesions present variable contrast enhancement and usually little or no mass effect ${ }^{7}$.

Clinically, this pattern of involvement was associated with a neurodegenerative syndrome of highly variable severity and course. The symptoms range from subtle tremor to profound disabilities, including ataxia, dysarthria, and psychomotor deterioration ${ }^{3}$.
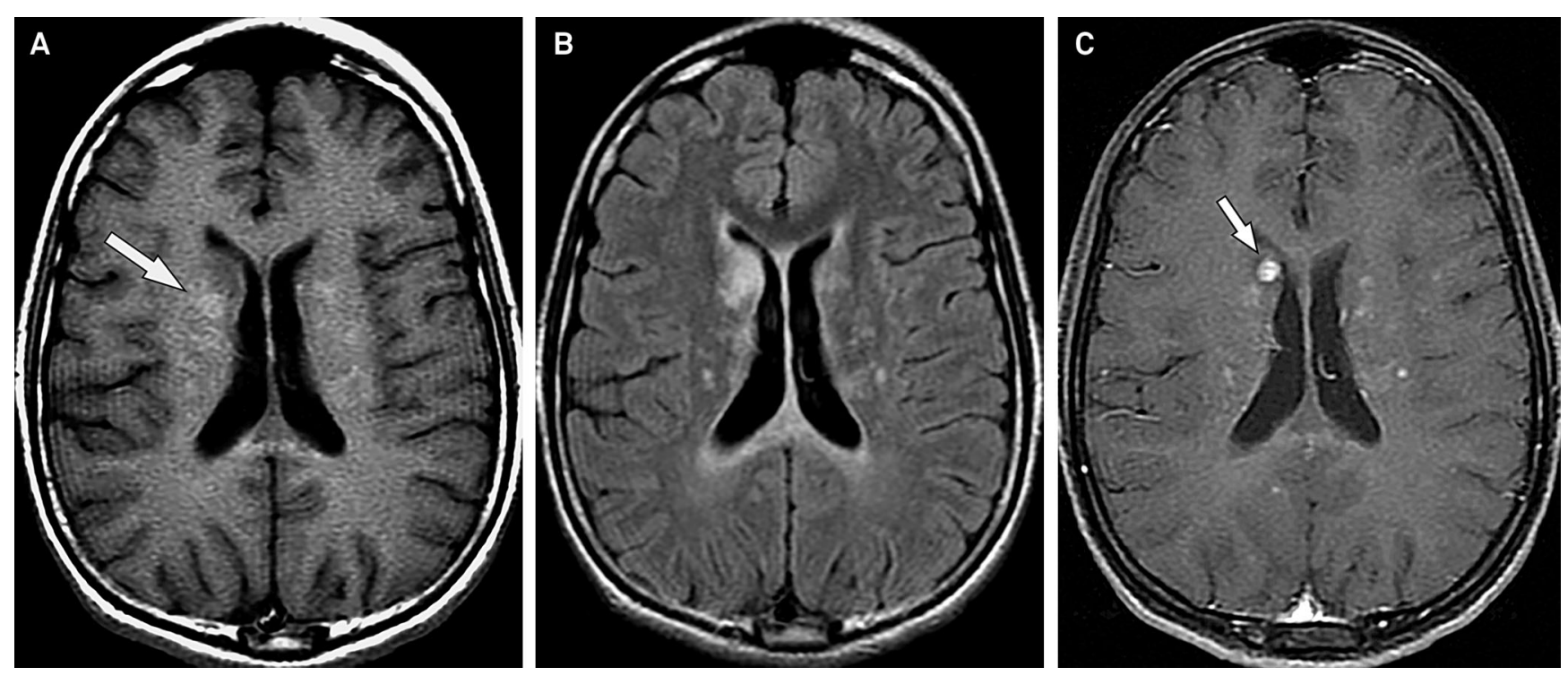

Figure 6. The same patient as in Figure 5, images obtained at the basal ganglia level. Axial T1WI demonstrates that these lesions are hyperintense (A and arrow). They are also hyperintense in a corresponding FLAIR image (B); and some of them clearly present enhancement in a postcontrast axial T1WI at the same level ( $\mathrm{C}$ and arrow). 
The MR signal intensity abnormalities in ND-LCH are not pathognomonic for LCH. Similar findings can be observed in the cerebellum of patients with cerebrotendinous xanthomatosis, Erdheim-Chester disease, corticobasal degeneration, or toxic demyelination. Symmetric hyperintensities on T1-weighted images were also observed in the basal ganglia of patients with chronic liver failure and in patients with long-term parenteral nutrition ${ }^{10}$.

The white matter changes consist of two other patterns: a perivascular pattern and a leukoencephalopathy pattern ${ }^{3}$. Dilated Virchow-Robin spaces are best seen on T2WI and can be barely visible with a width of approximately $2 \mathrm{~mm}$. The role of Virchow-Robin spaces in the pathophysiology of CNS-LCH remains to be investigated. No biopsy samples to confirm histopathology are available from such lesions, but they might be consistent with either an active inflammatory process or the sequelae of an inflammatory process following the distribution of the perivascular spaces ${ }^{2}$.

The leukoencephalopathy-like pattern involves the cerebellar white matter, the pons, and the periventricular white matter and presents with symmetric patchy areas characterized by high signal intensity on T2WI and low signal intensity on TIWI without a clear vascular distribution ${ }^{7}$. In most cases, supratentorial lesions were confluent and predominantly periventricular, with blurred margins. Breakdown of the blood brain barrier were only seen in a few severely disabled patients ${ }^{3}$.

Differential diagnosis includes acute disseminated encephalomyelitis, acute multiphasic disseminated encephalitis, metabolic and degenerative disorders, or chemo-/radiation therapy sequelae ${ }^{7}$.

Atrophic changes may be limited to the cerebellar hemispheres but may also be global, usually in patients with a progressive symptomatic course ${ }^{11}$.

\section{ROSAI DORFMAN DISEASE}

Rosai-Dorfman disease, also called 'sinus histiocytosis with massive lymphadenopathy' (SHML), most commonly affects young adults - males more often than females with a peak age around 20 years. It is a sporadic disease with occasional clusters. Etiology is unknown. The lesional cell derivation from circulating mononuclear cells suggests a reactive disorder and the increased incidence of serum auto-immune antibodies during active disease suggests a possible pathogenic correlation with an immune regulatory process compromise. HHV-6 and Epstein-Bar viruses have been implicated in some cases ${ }^{12}$.

\section{Clinical features}

In its classic form, Rosai-Dorfman disease causes a massive, painless cervical lymphadenopathy, fever, weight loss and an elevated erythrocyte sedimentation rate. It is the presenting symptom in the vast majority (approximately $90 \%$ ) of cases. Inguinal and axillary lymphadenopathies are less common (20\%). Other sites of lymph node enlargement involvement include mediastinum and upper para-aortic nodes $(10-15 \%)^{13}$.

Extranodal sites are involved simultaneously in about $43 \%$ of cases and isolated extranodal RDD occurs in only $23 \%$ cases. Irrespective of concurrent lymph node involvement, the most frequent extranodal sites in decreasing order are skin/soft tissue, nasal cavity/paranasal sinuses, orbit/eye/ocular adnexa, bone, salivary gland, CNS, oral cavity, kidney/genitourinary tract, respiratory tract, liver, tonsil, breast, gastrointestinal tract and heart. Hepatosplenomegaly is uncommon ${ }^{14}$.

In exceedingly rare circumstances, CNS can be affected. About $70 \%$ of intracranial cases had no lymphadenopathy and $52 \%$ had no associated systemic disease. So, the classic clinical presentation is not a requirement for the diagnosis. Some scholars believe that the usage of this term SHML in CNS is not quite appropriate because of the lack of lymphadenopathy in the great majority of cases. The mean age at onset is older in patients with intracranial RDD (mean age: 39.4 years) than in systemic disease with massive lymphadenopathy. The sex distribution is $1.5: 1$ (male: female) in isolated intracranial disease. Intracranial RDD causes a variety of symptoms, such as headache, seizure, numbness, paraplegia, cranial nerve deficits and visual disturbances depending on the location of the lesion (15). Symptoms of hypothalamic pituitary axis dysfunction such as diabetes insipidus were also described ${ }^{16}$.

The clinical course may be chronic or fluctuating but is usually self-limiting, with $70 \%$ to $80 \%$ of individuals having spontaneous resolution. The mortality rate is low but death may result from CNS or renal involvement, immune dysfunction, and opportunistic infections ${ }^{17}$.

\section{Histopathologic findings}

The definitive diagnosis of Rosai-Dorfman disease relies on a pathologic and immunohistochemical examination. RDD is characterized by abundant sheets of large- and medium-sized vacuolated histiocytes in a fibrous stroma, interspersed with foci of chronic inflammatory cells. There are numerous Russell bodies. A feature of the condition is the presence of lymphophagocytosis (emperipolesis), that is characteristic but not pathognomonic of RDD (in only $70 \%$ of cases), although this may be less marked in intracranial disease. The histiocytes typically stain positive for S100 and CD68 and negative for CDla. Both T and B lymphocytes are commonly present. The disease can be differentiated from LCH in which eosinophils are conspicuous, the histiocytes do not exhibit emperipolesis, contain Birbeck granules on electron microscopy, and are immunoreactive for CDla, these last two absent in $\mathrm{RDD}^{15,18,19}$. 


\section{MRI findings and differential diagnosis}

CNS involvement is unusual with or without nodal disease. The usual finding in this disease is an intracranial extraaxial mass, although intraparenchymal and intraventricular cases have been reported ${ }^{18}$.

Cases of spinal epidural or subdural masses are a rare manifestation of $\mathrm{RDD}^{20}$. Intraparenchymal brain and intramedullary spinal cord lesion are even rarer $^{21}$.

Intracranial involvement accounted for $75 \%$ and spinal canal involvement for 25\%. Frequent intracranial locations of RDD include the cerebral convexities, parasagittal and petroclival (Figure 7). Suprasellar region involvement is extremely rare ${ }^{16}$. There have also been reports of cavernous sinus invasion ${ }^{22}$.

Most lesions in intracranial Rosai-Dorfman disease mimic meningioma in both clinical and radiologic aspects (Figure 7), making the diagnosis more difficult. MRI reveals well-defined dural-based lesions, single or multiple, with hypointensity or isointensity on T1WI and T2WI, and with marked homogeneous enhancement after the administration of gadolinium. Some lesions are associated with adjacent bone erosion ${ }^{19}$.

Perfusion-weighted techniques disclose decreased values of relative cerebral blood volume inside the lesions. RosaiDorfman disease is essentially a lymphoproliferative disorder with no neoangiogenesis, as evidenced by histopathological findings. That feature might be important for differentiating the disease from meningiomas, hemangiopericytomas and solitary fibrous tumors of the meninges, which typically have markedly increased perfusion parameters. However, a recent report has shown that Rosai-Dorfman disease may also show increased perfusion due to its high positivity for CD34 and CD31 antibodies, which are surrogate markers for the intrinsic vascularization of lesions ${ }^{23}$.

The natural history of Rosai-Dorfman disease remains uncertain, although most cases are benign and self-limited. The spontaneous regression of Rosai-Dorfman disease was reported in systemic disease, but not in intracranial lesions. A few cases in the literature involved Rosai-Dorfman disease of the CNS, with the regression of nonsurgically treated lesions after the administration of corticosteroid agents ${ }^{19}$.

The excision of these lesions is normally undertaken to confirm the diagnosis and reduce the mass effect. Complete macroscopic excision is the goal of surgery, and when this is achieved patients have a normal life expectancy with no recurrent disease. Early and significant recurrence has been reported in rare cases, which implies that this disease process may not always follow an indolent course ${ }^{24}$.

\section{ERDHEIM-CHESTER DISEASE}

The earliest Erdheim-Chester disease (ECD) is a rare, sporadic, systemic, histiocytic disease of unknown etiology. It is pathologically characterized by xanthogranulomatous infiltrate and clinically and radiologically defined by bone involvement, which is a constant and pathognomic finding ${ }^{25}$. ECD generally affects the long bones of the lower limbs. Related radiographic findings are diffuse or patchy opacities of the tibia and/or fibula, medullary sclerosis and cortical thickening of the metaphyseal and diaphyseal portions without epiphyseal changes. On 99 mTc scintigraphy, intense,
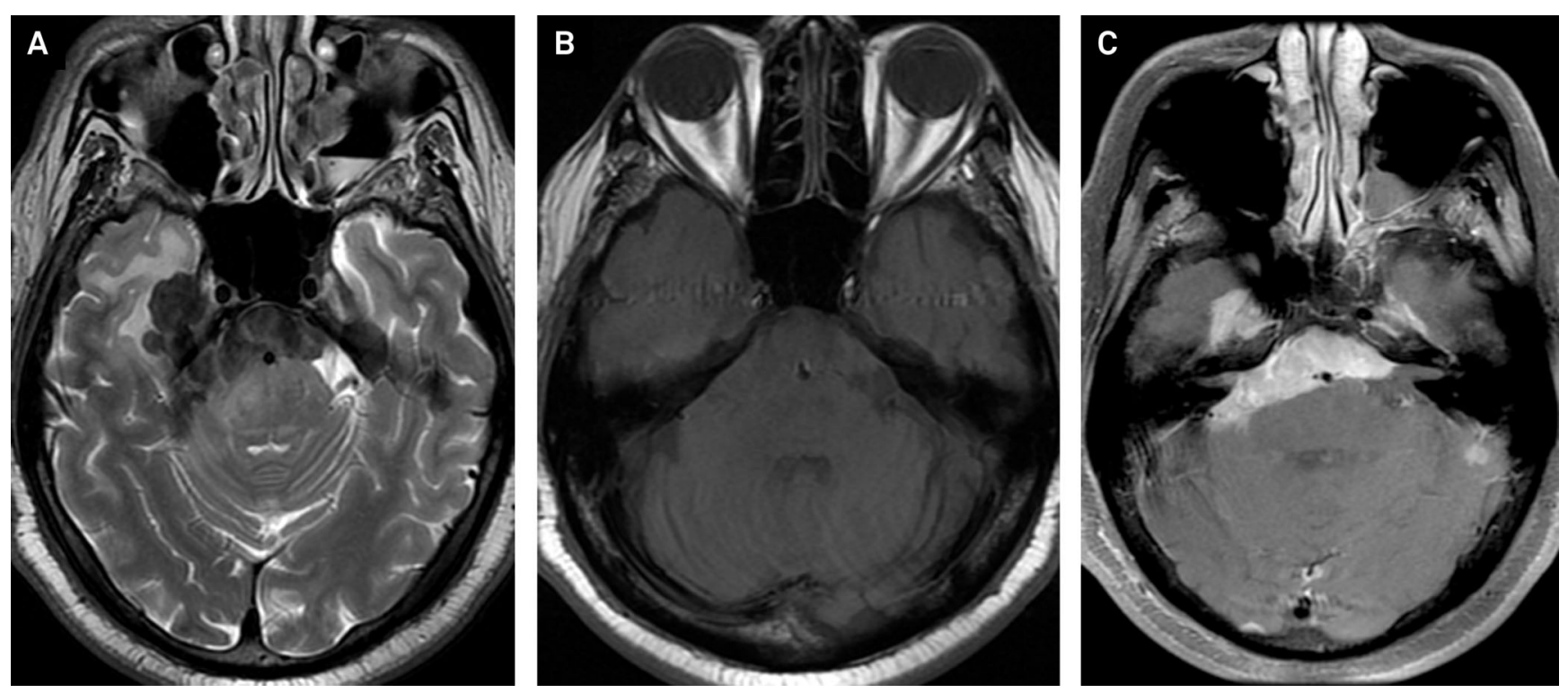

Figure 7. A 46-year-old patient diagnosed with Rosai Dorfman disease. Axial T2WI shows a large petroclival extra-axial lesion, with marked hypointensity, extending into the right temporal fossa, prepontine and right cerebellopontine cisterns (A). There is mass effect on the corresponding temporal lobe and brainstem, with associated vasogenic edema. Axial T1WI demonstrates isointensity (B). Postcontrast axial T1WI depicts marked enhancement of the mass (C). 
bilateral and symmetrical tracer uptake by the long-bone diaphyses and metaphyses are revealed, reflecting increased osteoblastic activity in those regions ${ }^{26}$.

\section{Clinical features}

Erdheim-Chester disease affects both genders, though with a slight male predilection. It has a peak incidence between the fifth and sixth decade of life, although it may manifest at any age. ECD is a progressive disease that has a 5 -year survival rate of $41 \%^{26}$. The most common presenting symptom is bone pain, fever and weight loss. Physical examination reveals no consistent findings; however, hypertension has commonly been noted. Laboratory evaluation also tends to reveal unremarkable findings; however, abnormal levels of neutral fat, free fatty acids, and triglycerides have been reported ${ }^{27}$.

About half of all patients have extraskeletal manifestations. The disease can affect multiple organs systems, including musculoskeletal, CNS, pulmonary and renal systems. Retroperitoneal involvement is secondary to infiltration of the fat and surrounding structures by histiocytes and associated fibrosis causing renal impairment. The cardiovascular manifestations of ECD have occurs in about $40 \%$ of the patients with periaortic "fibrosis" as the most frequent cardiovascular involvement. Other manifestations include heart failure, valvular dysfunction, renovascular hypertension and pericarditis. The pulmonary involvement manifests as dyspnea or a dry cough. Most patients have mediastinal infiltration, diffuse interstitial infiltrates and pleural and/or interlobar septal thickening best seen on high-resolution computed tomography ${ }^{28}$.

Alterations of the skin and mucosae and nodal involvement are present in ECD, even though it tends to be more extensive in $\mathrm{LCH}^{27}$. The cutaneous involvement show xanthomas and xanthelasmas, more commonly present in the patients with severe hyperlipidemia ${ }^{25}$.
In ECD the most frequent CNS symptoms and signs are central diabetes insipidus that may be the initial symptom of ECD and may be the only one for many years. Next in reported frequency are cerebellar symptoms and signs, with ataxia usually of gait ${ }^{29}$. Neurologic symptoms are reported in about one-third of ECD cases. Signs and symptoms such as pyramidal syndromes, seizures, headaches, neuropsychiatric or cognitive symptoms, sensory disturbances, cranial nerve paralysis or even asymptomatic lesions were also reported ${ }^{30}$.

The prognosis depends on the extent and distribution of the extraskeletal manifestations. Respiratory distress, extensive pulmonary fibrosis, and cardiac failure are the most common causes of death ${ }^{31}$.

\section{Histopathologic findings}

ECD is characterized by a mononuclear infiltrate consisting of lipid laden, foamy histiocytes ${ }^{31}$. In contrast to Langerhans histiocytosis, lesions of ECD are populated by histiocytes without nuclear grooves that show CD68 but little or no S100 and no CDla immunoreactivity. Birbeck granules are also absent ${ }^{32}$. However, these two diseases may coexist, and cases of double infiltration with mixed $\mathrm{LCH}$ and ECD have been reported ${ }^{33}$.

\section{MRI findings and differential}

CNS involvement may present as involvement of the hypothalamic-pituitary axis, brain parenchyma, meninges or vessels. The HPR manifests as loss of high signal intensity of the posterior pituitary lobe on T1WI. Another possible finding ranges from pituitary stalk thickening to a rare tumorlike mass that can extend to the hypothalamus.

Intraparenchymal involvement is very uncommon and is evident on MRI as multifocal, diffuse, or infiltrative lesions ${ }^{34}$. These lesions are mainly verified at the infratentorial compartment (Figure 8), including the brainstem, cerebellum, and middle cerebellar peduncles, and rarely in the cerebral
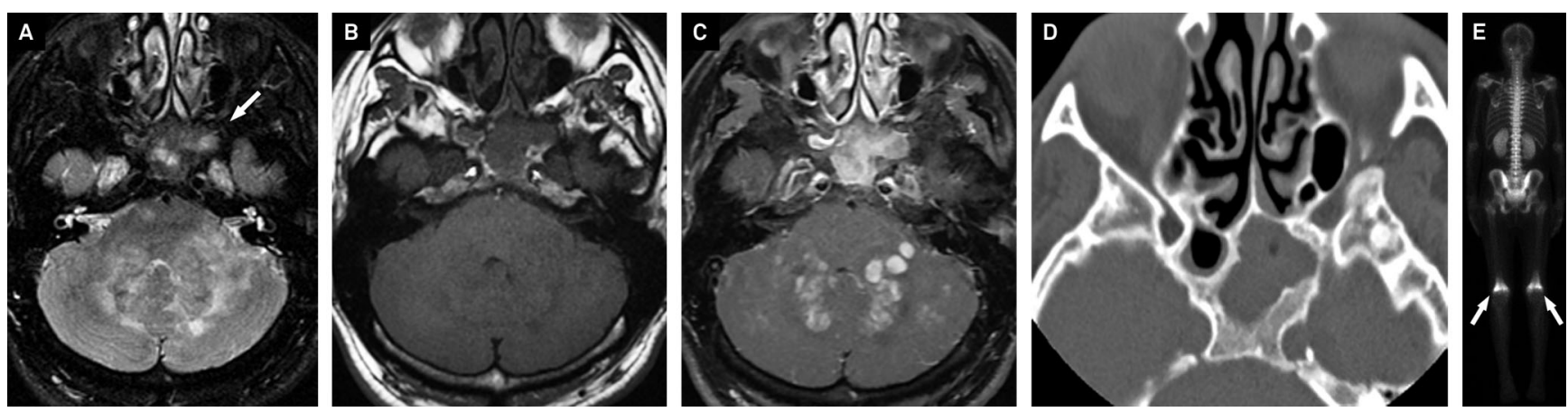

Figure 8. A 35-year-old male patient diagnosed with Erdheim-Chester disease. Axial T2WI shows bilateral hyperintensity in the cerebellar white matter, middle cerebellar peduncles and pons, as well as low signal intensity filling the sphenoid sinus (A and arrow). Axial T1WI reveals that the lesions are hypointense (B). Gadolinium-enhanced axial T1WI shows enhancement of the lesions and inside the sphenoid sinus (C). Corresponding axial CT image from a paranasal sinus study reinforces the filling of the sphenoid sinus associated to the presence of thickened sclerotic walls (D). Bone scintigraphy reveals symmetrically increased radionuclide uptake at the level of the distal femoral diaphyses and metaphyses, a quite distinctive finding of the disease (E). 
hemispheres ${ }^{30,35,36}$. The dentate nucleus may show abnormal symmetric high signal intensity on T2WI that can also be found in the middle cerebellar peduncles. These lesions present variable contrast enhancement and usually little or no mass effect. If the exact origin of these nongranulomatous lesions remains unknown, histological examination of these dentate nuclei lesions can be performed to reveal extensive loss of myelin sheath tissue, with gliosis and marked sparing of axons ${ }^{35}$.

Meningeal lesions and meningioma-like mass lesions: either a single/multiple dural mass, or diffuse pachymeningeal thickening, or even both of those $e^{32,35}$. Parasellar lesions have also been reported, which may infiltrate the cavernous sinus $^{22}$. Although Erdheim-Chester disease rarely affects spine, it should still be considered in the differential diagnosis of subdural or epidural masses ${ }^{37}$.

Intracranial periarterial or intravenous infiltrations in ECD are exceedingly rare, but it may be overlooked because they can be asymptomatic. These infiltrations should be detected before catastrophic symptoms related to stroke develop. Lesions in venous sinuses are extremely rare, which may be occlusive ${ }^{35}$.

At MRI, these intracranial masses often had low signal intensity on T2WI, an isointense signal on T1WI, and intense homogeneous enhancement on gadoliniumenhanced T1WI. Intraparenchymal masses may show, less frequently, a hyperintense signal on $\mathrm{T}_{2} \mathrm{WI}^{35}$. The persistent gadolinium enhancement is a unique distinguishing feature of Erdheim-Chester disease ${ }^{27}$. Tien et al. ${ }^{38}$ first reported the occurrence of persistent enhancement of intracerebral masses, after 8 days of administration of gadolinium-based contrast medium. The precise mechanism is unknown, but it could be characteristic of abnormal gadolinium retention by histiocytes ${ }^{36}$. Some reports demonstrate persistent enhancement for 2 or more weeks after intravenous GdDTPA administration ${ }^{32}$. However, although such retention is seen only in Erdheim-Chester histiocytic lesions, it is not a universally observed feature ${ }^{27}$.

The association of intracranial lesions with sinus walls osteosclerosis with low signal intensity filling and/or orbital masses are frequent, showing facial and/or skull bone thickening. Retro-orbital involvement is visualized as either diffuse masses surrounding the optic nerves or diffuse infiltration of the retro-ocular fat, associated with exophthalmia. These orbital lesions show the same image characteristics seen on the intracranial lesions ${ }^{35}$.

\section{HEMOPHAGOCYTIC LYMPHOHISTIOCYTOSIS}

Hemophagocytic lymphohistiocytosis (HLH) is a rare clinical syndrome characterized by severe hyper-inflammation caused by uncontrolled proliferation and infiltration of activated lymphocytes and histiocytes. High amounts of inflammatory cytokines are secreted and multiple organs are affected, including liver, spleen, lymph nodes, bone marrow and the $\mathrm{CNS}^{39}$. This disease, a non-Langerhans cell histiocytosis, also known as hemophagocytic syndrome ${ }^{40}$, may be a medical emergency and might lead to a rapid fatal outcome if not treated promptly and adequately ${ }^{41}$.

Historically, two forms of HLH are distinguished: genetic and acquired forms. The genetic (primary) is inherited in an autosomal recessive or X-linked manner and can be divided into two subgroups: a) familial HLH (FHLH) in which the clinical syndrome of HLH is the only manifestation; and b) the immune deficiencies Chédiak-Higashi syndrome, Griscelli syndrome and X-linked proliferative syndrome, which have distinctive clinical features besides the sporadic, though frequent development of HLH. The FHLH usually occurs in early infancy in 70-80\% of cases, often below one year of age, and it is found especially in ethnic groups where consanguineous marriages are common ${ }^{39,42,43}$.

Acquired (secondary) HLH can occur in all age groups. It occurs as a reactive process in response to infective agents as well as to malignancies and tends to occur in immunocompromised individuals ${ }^{44}$. Leading triggering agents in infection-associated hemophagocytic syndrome are viruses of the herpes group, especially Epstein-Barr and cytomegalovirus. Lymphoma has been reported mostly in adults. A frequent non-viral agent associated with HLH in children is Leishmania $^{43,45}$.

The presence of an infection was originally thought to discriminate between familial and acquired forms. However, it is now clear that most episodes in the genetic forms of HLH are also trigged by infections ${ }^{39}$.

\section{Clinical features and diagnostic criteria}

Until recently, it was widely believed that symptoms of familial hemophagocytic lymphohistiocytosis generally arose during infancy and early childhood. With the more widespread availability of genetic testing, it is apparent that the first significant episode of FHLH can occur throughout life, including in utero.

In the most typical form of FHLH, the clinical course is characterized by prolonged fever and hepatosplenomegaly. Although the clinical presentation of HLH is not neurological in most of the patients, a puzzling neurological onset may be observed in about $20 \%$ of the cases and up to $73 \%$ of affected children ultimately develop CNS involvement.

Neurologic symptoms may dominate the initial clinical course with seizures and/or ataxia. Neurologic findings may be highly variable and can include irritability, hypo or hypertonia, cranial nerve palsies, meningismus, signs of increased intracranial pressure and altered mental status.

Rash, lymphadenopathy and diarrhea are less frequently observed. In the early days to months of the disease, 
symptoms may improve spontaneously, followed by clinical exacerbations. It is important to say that hemophagocytosis may not be obvious on bone marrow biopsy examination early in the course of the disease $\mathrm{e}^{41,46,47}$.

The main problem is that initially HLH clinical presentation can mimic a normal infection and too little attention is dedicated to unusually marked hepatosplenomegaly or unusually severe blood count changes. Much time then may be lost with extensive work-up for an infectious disease or with prolonged antibiotic treatment. Ferritin, triglycerides, and fibrinogen are not routinely determined in children with fever; HLH has to be kept in mind to order these laboratory studies. Also progression of symptoms may be overlooked or may not be considered unusual. The absence of hemophagocytosis is often the reason why the diagnosis of HLH is ruled out. Another problem is that the identification of an infectious organism may serve to explain all symptoms, without taking into account that its severity and progression indicates an immune system deficiency ${ }^{39}$.

In 1991 the familial haemophagocytic lymphohistiocytosis Study Group of the Histiocyte Society proposed diagnostic guidelines for HLH. They include: fever of \pm 7 days duration, splenomegaly, cytopenia affecting at least two cell lines (hemoglobin $<9.0 \mathrm{~g} / \mathrm{dL}$, platelets $<100 \times 10^{9} / \mathrm{L}$, neutrophils $\left.<1.0 \times 10^{9} / \mathrm{L}\right)$, hypofibrinogenemia $(<1.5 \mathrm{~g} / \mathrm{L})$ and/or hypertriglyceridemia $(>2 \mathrm{mmol} / \mathrm{l})$ as well as hemophagocytosis in bone marrow or other tissues ${ }^{48}$.

\section{Histopathologic findings}

Pathologic examination of affected organs shows an infiltration of benign-appearing histiocytes with hemophagocytosis, predominantly red blood cells and neutrophils. Bone marrow is the tissue most widely used for diagnostic examination, and bone marrow smears may best demonstrate hemophagocytosis. Lymph nodes and spleen may reveal profound generalized lymphoid depletion with sinusoidal infiltration by hemophagocytic histiocytes in sinusoids. FHLH may express a characteristic phenotype exhibiting CD11b, CD21, CD25, CD30, CD 36 and S100 positivity. In most cases of secondary HLH EBV was found to be the infectious agent and atypical lymphocytes (with are the hallmark of acute EBV infection) are absent or diminished, reflecting a decrease in the number of activated $\mathrm{CD} 8+\mathrm{T}$ cells normally seen in response to $\mathrm{EBV}^{49}$.

\section{MRI findings and differential diagnosis}

The neurological manifestations are variable and in the same way the imaging studies do not demonstrate any specific feature. This can delay the diagnosis, with a high risk of a sudden severe deterioration and even death ${ }^{41}$.

Characteristic findings of HLH on brain MRI include multifocal lesions with or without mass-like lesions that can be observed in the subcortical areas in the cerebral hemispheres and cerebellum. They may show restricted diffusion, contrast enhancement (leptomeningeal, nodular or peripheral enhancement) (Figures 9 and 10). Some bleeding may occur. Diffuse white matter lesions are less common ${ }^{40,50}$.

Hypoxic ischemic disease, acute disseminated encephalomyelitis, cerebrotendinous xanthomatosis, other histiocytosis, mild encephalitis/encephalophathy with reversible splenium lesion (MERS) are some disease that may be included in the differential diagnosis ${ }^{40,41}$.

\section{FINAL REMARKS}

Histiocytosis is a group of rare disease with some peculiarities that help to differentiate each one of them. Sometimes,
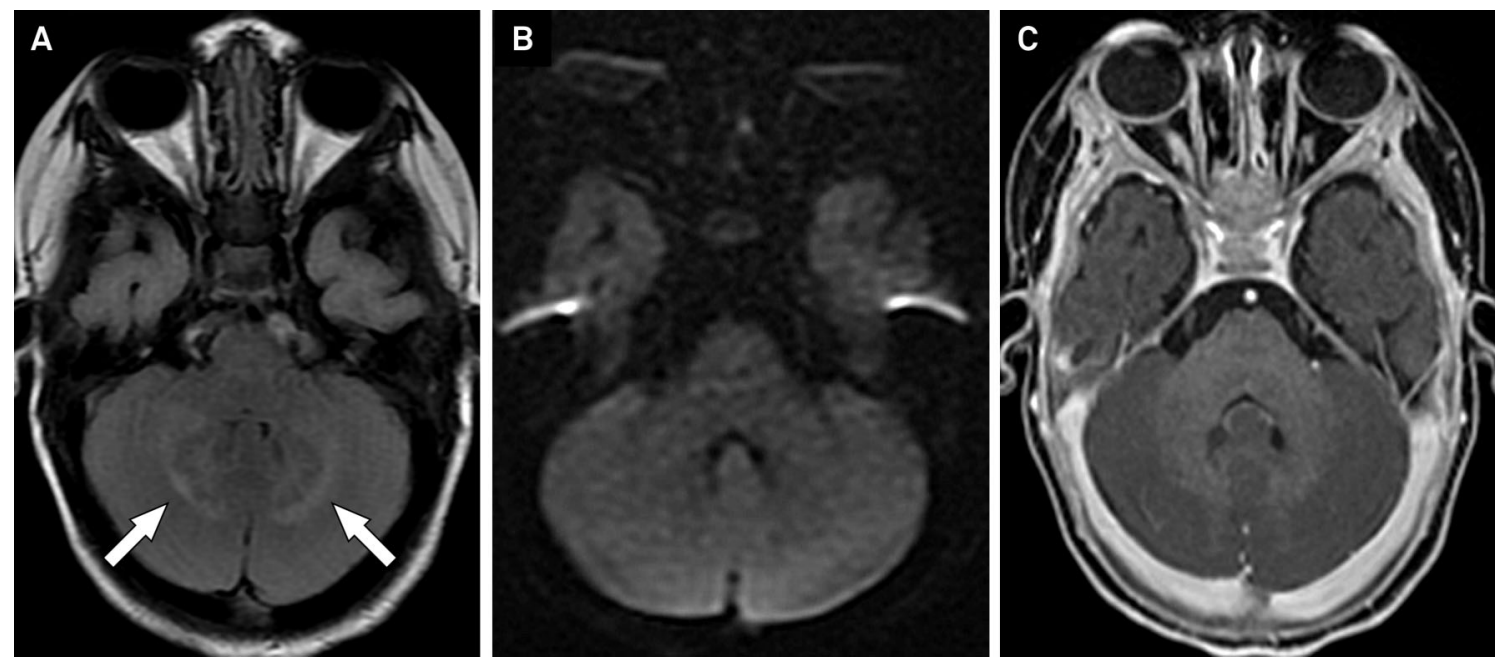

Figure 9. Patient with 6 months of life presenting bilateral paresis of the sixth cranial nerve, hepatomegaly, increased levels of ferritin and triglycerides, hypofibrinogenemia and pancytopenia, diagnosed as hemophagocytic lymphohistiocytosis. Axial FLAIR (A), diffusion-weighted (B) and enhanced T1WI (C) show subtle hyperintense symmetrical deep cerebellar white matter lesions without restricted diffusion or gadolinium enhancement. 

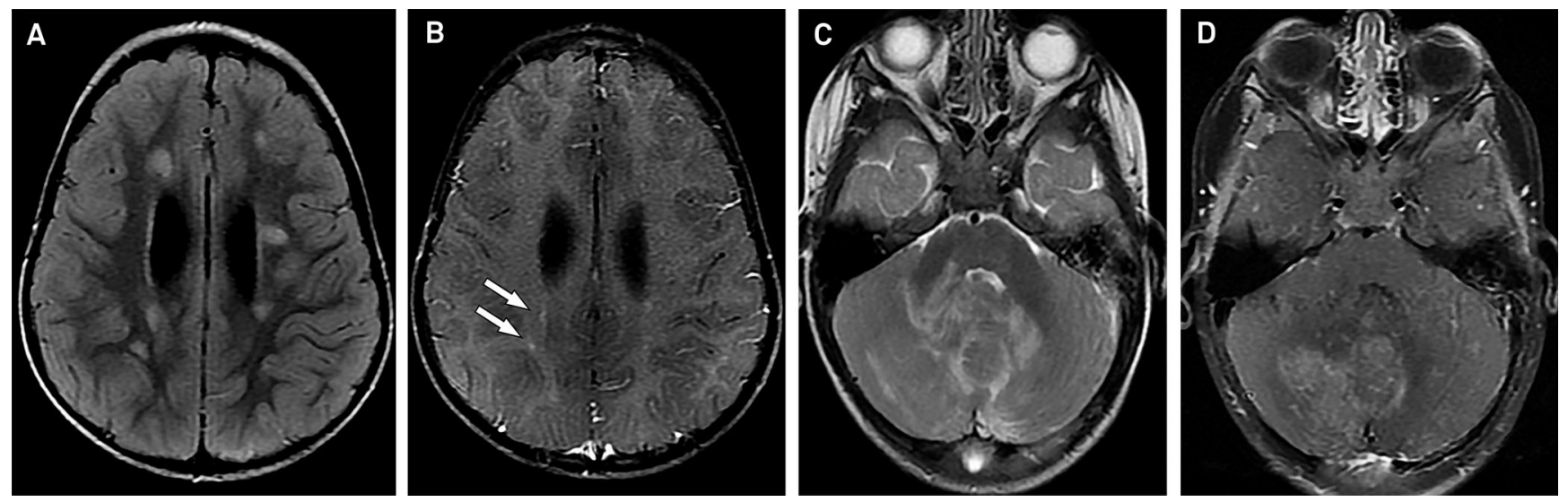

Figure 10. Two-year-old patient with hemophagocytic lymphohistiocytosis. Axial FLAIR (A) and postcontrast T1WI (B) show lesions in the deep and periventricular white matter of the cerebral hemispheres, some of them associated to contrast enhancement (arrows in B). Axial T2WI (C) and corresponding postcontrast T1WI (D) demonstrate extensive involvement of cerebellar hemispheres and vermis, extending to the right middle cerebellar peduncle, associated to mass effect and heterogeneous contrast enhancement.

however, imaging picture can be similar and some overlap in MRI findings might occur. Some of the most common findings, like hypothalamic-pituitay axis involvement, meningeal and meningioma-like mass lesions, choroid plexus lesions, and intraparenchymal involvement ( for example, high signal intensity on T1-weighted MRI images in cerebellum and basal ganglia) must be recognized to include this possibility in the differential diagnosis, whenever considered pertinent.

\section{References}

1. The French Langerhans' Cell Histiocytosis Study Group. A multicentre retrospective survey of Langerhans' cell histiocytosis: 348 cases observed between 1983 and 1993. Arch Dis Child 1996;75:17-24

2. Grois N, Fahrner B, Arceci RJ, et al. Histiocyte Society CNS LCH Study Group. Central Nervous System Disease in Langerhans Cell Histiocytosis. J Pediatr 2010;156:873-881.

3. Prayer D, Grois N, Prosch H, Gadner H, Barkovich AJ. MR imaging presentation of intracranial disease associated with Langerhans cell histiocytosis. AJNR Am J Neuroradiol 2004;25:880-891.

4. Grois N, Prayer D, Prosch H, et al. Neuropathology of CNS disease in Langerhans cell histiocytosis. Brain 2005;128:829-838.

5. Arico M, Egeler RM. Clinical aspects of Langerhans cell histiocytosis. Hematol Oncol Clin North Am 1998;12:247-258.

6. Grois NG, Favara BE, Mostbeck GH, et al. Central nervous system disease in Langerhans cell histiocytosis. Hematol Oncol Clin North Am 1998;12:287-305.

7. Kalnins AU, Mansour A, Castillo M. Neuroradiologic imaging findings in histiocytosis syndromes in children and young adults. Neurographics 2012;2:106-118.

8. Maghnie M, Cosi G, Genovese E, et al. Central diabetes insipidus in children and young adults. N Engl J Med 2000;343:998-1007.

9. Grois N, Prosch H, Waldhauser F, et al. Pineal gland abnormalities in Langerhans cell histiocytosis. Pediatr Blood Cancer 2004;43:261-266.

10. Prosch H, Grois N, Wnorowski M, et al. Long-term MR imaging course of neurodegenerative Langerhans cell histiocytosis. AJNR Am J Neuroradiol 2007;28:1022-1028.

11. Grois N, Barkovich AJ, Rosenau W, Ablin AR. Central nervous system disease associated with Langerhans' cell histiocytosis. AmJ Pediatr Hematol Oncol 1993;15:245-254.

12. Henter JI, Tondini C, Pritchard J. Histiocyte disorders. Crit Rev Oncol Hematol 2004;50:157-174.
13. Rosai J, Dorfman RF. Sinus histiocytosis with massive lymphadenopathy: a pseudolymphomatous benign disorder. Analysis of 34 cases. Cancer 1972;30:1174-1188

14. Gaitonde, S. Multifocal extranodal sinus histiocytosis with massive lymphadenopathy. Arch Pathol Lab Med 2007;131:1117-1121.

15. Zhu H, Qiu LH, Dou YF, Wu JS, Zhong P, Jiang CC, Xu R, Wang XQ Imaging characteristics of Rosai-Dorfman disease in the central nervous system. Eur J Radiol 2012;81:1265-1272.

16. Lou X, Chen Z-y, Wang F-L, Ma L. MR findings of Rosai-Dorfman disease in sellar and suprasellar region. Eur J Radiol 2012,81:1231-1237.

17. Andriko J-A W, Morrison A, Colegial CH, Davis BJ, Jones RV. RosaiDorfman Disease isolated to the central nervous system: a report of 11 cases. Mod Pathol 2001;14:172-178.

18. Gupta DK, Suri A, Mahapatra AK, et al. Intracranial Rosai-Dorfman disease in a child mimicking bilateral giant petroclival meningiomas: a case report and review of literature. Childs Nerv Syst 2006;22:1194-1200.

19. Ramos AA, Vega MAA, Alles JVD, Garcia MJA, Martinez AM. Multiple involvement of the central nervous system in Rosai-Dorfman disease. Pediatr Neurol 2012;46:54-56.

20. Bhandari A, Patel PR, Patel MP. Extranodal Rosai-Dorfman disease with multiple spinal lesions:a rare presentation. Surg Neurol 2006;65:308-311.

21. Jurić G, Jakić -Razumović J, Rotim R, Zarković K. Extranodal sinus histiocytosis (Rosai-Dorfman Disease) of the brain parenchyma. Acta Neurochir 2003;145:145-149.

22. Razek AA, Castilho M. Imaging lesions of the cavernous sinus. AJNR 2009;30:444-452.

23. Hingwalaa D, Neelimab R, Kesavadasa C, Thomasa B, Kapilamoorthya TR, Radhakrishnanb V. Advanced MRI in RosaiDorfman disease: Correlation with histopathology. J Neuroradiol 2011;38:113-117. 
24. Rocco F, Garnett MR, Puget S, et al. Cerebral localization of RosaiDorfman disease in a child. J Neurosurg 2007;107:147-151.

25. Olmos SJM, Canga A, Velero C, Gonzalez-Macias J. Imaging of Erdheim-Chester disease. J Bone Min Res 2002;17:381-383.

26. De Filippo M, Ingegnoli A, Carloni A, et al. Erdheim-Chester disease: clinical and radiological findings. Radiol Med 2009;114:1319-1329.

27. Martinez R. Erdheim-Chester disease: MR of intraaxial and extraaxial brain stem lesions. AJNR 1995;16:1787-1790.

28. Pan A, Doyle T, Schlup M, Lubcke R, Schultz M. Unusual manifestation of Erdheim-Chester disease. BMC Gastroenterol 2011;11:77.

29. Wright RA, Hermann RC, Parisi JE. Neurological manifestations of Erdheim-Chester disease. J Neurol Neurosurg Psychiatry 1999;66:72-75

30. Lachenal F, Cotton F, Desmurs-Clavel H, et al. Neurological manifestations and neuroradiological presentation of ErdheimChester disease: report of 6 cases and systematic review of the literature. J Neurol 2006; 253(10):1267-1277.

31. Sheu S-Y, Wenzel RR, Kersting C, Merten R, Otterbach F, Schmid KW. Erdheim-Chester disease: case report with multisystemic manifestations including testes, thyroid, and lymph nodes, and a review of literature. J Clin Pathol 2004;57:1225-1228.

32. Johnson MD, Aulino JP, Jagasia M, Mawn LA. Erdheim-Chester Disease mimicking multiple meningiomas syndrome. AJNR 2004;25:134-137.

33. Vital C, Bioulac-Sage P, Tison F, et al. Brain stem infiltration by mixed Langerhans cell histiocytosis and Chester-Erdheim disease: more than just an isolated case? Clin Exp Pathol 1999;47:71-76.

34. Oweity T, Scheithauer BW, Ching HS, Lei C, Wong KP. Multiple system Erdheim-Chester disease with massive hypothalamic-sellar involvement and hypopituitarism. J Neurosurg 2002;96:344-351.

35. Drier A. Cerebral, Facial, and Orbital Involvement in Erdheim-Chester disease: CT and MR imaging findings. Radiology 2010;255:586-594.

36. Adem C, Hélie O, Lévêque C, Taillia H, Yves-Sébastien Cordoliani Y-S. Erdheim-Chester disease with central nervous system involvement. Radiology 2005;234:111-115.

37. Albayram S, Kizilkilic O, Zulfikar Z, Islak C, Kocer N. Spinal dural involvement in Erdheim-Chester disease: MRI findings. Neuroradiology 2002;44:1004-1007.
38. Tien RD, Brasch RC, Jackson DE, Dillon WP. Cerebral ErdheimChester disease: persistent enhancement with Gd-DTPA on MR images. Radiology 1989;172:791-792.

39. Janka G.E . Familial and acquired hemophagocytic lymphohistiocytosis. Eur J Pediatr 2007;166:95-109.

40. Ishikura R, Ando K, Hirota S, Okamoto N, Fatterpekar G, Sacher M. Callosal and diffuse white matter lesions with restricted water diffusion in hemophagocytic syndrome. Magn Reson Med Sci 2010;9:91-94.

41. Chiapparini L, Uziel G, Vallinoto C, et al. Hemophagocytic lymphohistiocytosis with neurological presentation: MRI findings and a nearly miss diagnosis. Neurol Sci 2011;32:473-477.

42. Decaminada N, Cappellini M, Mortilla M, et al. Familial hemophagocytic lymphohistiocytosis: clinical and neuroradiological findings and review of the literature. Childs Nerv Syst 2010;26:121-127.

43. Janka GE. Hemophagocytic syndromes. Blood Rev 2007;21:245-253.

44. Ozgen B, Karli-Oguz K, Sarikaya B, Tavil B, Gurgey A. DiffusionWeighted Cranial MR imaging findings in a patient with hemophagocytic syndrome. AJNR 2006;27:1312-1314.

45. Gagnaire MH, Galambrun C, Stephan JL. Hemophagocytic syndrome: a misleading complication of visceral leishmaniasis in children - a series of 12 cases. Pediatrics 2000;106:58.

46. Filipovich, AH. Hemophagocytic lymphohistiocytosis $(H L H)$ and related disorders. Hematology Am Soc Hematol Educ Program 2009:127-131.

47. Fitzgerald NE, McClain KL. Imaging characteristics of hemophagocytic lymphohistiocytosis.Pediatr Radiol 2003;33:392-401.

48. Janka GE, Schneider EM. Modern management of children with haemophagocytic lymphohistiocytosis. Br J Haematol 2004;124:4-14.

49. Rezk SA, Sullivan JL, Woda BA. Nonneoplastic histiocytic proliferations of lymph nodes and bone marrow. In: Jaffe ES, Harris NL, Vardiman J, Campo E, Arber D (Eds). Hematopathology. Saunders/ Elsevier, Philadelphia; 2012:803-807.

50. Egmond ME, Vermeulen RJ, Peeters-Scholte CMPCD, et al. Familial hemophagocytic lymphohistiocytosis in a pediatric patient diagnosed by brain magnetic resonance imaging. Neuropediatrics 2011;42:191-193. 